\title{
Comparison of CGRP distributions in the maxillary sinus and trigeminal ganglion between elderly dentulous and edentulous humans
}

\author{
Hiroyuki Matsuda, ${ }^{1}$ Iwao Sato, ${ }^{1}$ Rieko Asaumi, ${ }^{2}$ Takuya Omotehara, ${ }^{1}$ Shinichi Kawata, ${ }^{1}$ Kenta Nagahori, ${ }^{1}$ \\ Zhong-Lian Li, ${ }^{1}$ Masahiro Itoh ${ }^{1}$ \\ ${ }^{1}$ Department of Anatomy, Tokyo Medical University, Tokyo \\ ${ }^{2}$ Department of Oral and Maxillofacial Radiology, School of Life Dentistry at Tokyo, The Nippon Dental University, \\ Tokyo, Japan
}

\begin{abstract}
Thickening of the Schneiderian membrane (SM, mucosa of the maxillary sinus) appears in the paranasal sinus. Information on SM thickening is available for patients receiving sinus lift treatments, which is a risk factor for SM excretory dysfunction. However, more information is needed on the structure of the SM and the relationship between the maxilla sinus and palatine with the alveolar bone and the SM for dental implant treatment in the human maxilla. One hundred twenty-six sides of the maxilla from 71 cadavers were subjected to cone-beam computed tomography analysis and macroscopic and immunohistochemical observations in this study. A thickened SM was mainly observed in the middle region of the basal layer of the maxillary sinus (MS). Strong calcitonin gene-related peptide (CGRP)-positive reactions were observed in the alveolar bone, oral mucosa, mucosa of the MS, and trigeminal ganglion (TG) cells in dentulous samples compared with edentulous samples. TG cells play important roles in delivering CGRP through axons to the mucosal gland and in regulating the maxilla-related thickening of the SM. These data could help determine CGRP functions in the mucosal gland and bone formation between dentulous and edentulous samples and indicate that CGRP may pass from the TG to the MS glands.
\end{abstract}

Key words: Mucosa; maxillary sinus; cone-beam computed tomography; immunohistochemistry; calcitonin gene-related peptide; trigeminal ganglion.

Correspondence: Iwao Sato, Department of Anatomy, Tokyo Medical University, 6-1-1, Shinjuku, Shinjuku-ku, Tokyo, 160-8402, Japan. Tel. +81.333516141 - Fax: +81.333411137. E-mail: iwaoa1@tokyo-med.ac.jp

Contributions: All authors provided substantive intellectual contributions and performed parts of the experiments. All authors have read and approved the final version of the manuscript and have agreed to be accountable for all aspects of the work.

Conflict of interest: The authors declare that they have no competing interests, and all authors confirm the accuracy.

Availability of data and materials: The data used to support the findings of this study are available from the corresponding author on reasonable request.

Ethical Approval: This study was performed in accordance with the principles of the Declaration of Helsinki (as revised in 2013). The study was approved by the Ethics Review Committee of Nippon Dental University (2018/21/July; no. NDU-T2015-20) and Tokyo Medical University, Institutional Review Board (2019/6/Feb.; TMU, no. T2018-0042). The cadavers used in the present study were donated to Tokyo Medical University and Nippon Dental University, Tokyo, Japan, based on the Act on Body Donation for Medical and Dental Education. All the donors willingly signed a form consenting to the body donation for education and research, and all donors could revoke the intended donation any time without any disadvantages. The use of cone-beam computed tomography data and materials of all cadavers was given permission by the individuals before donation and by their families according to the Law Concerning Cadaver Dissection and Preservation that was enacted in Japan in 1949.

Funding: This research received no specific grant from any funding agency in the public, commercial, or not for profit sectors. 


\section{Introduction}

The glandular tissues in paranasal sinuses, such as the maxillary, frontal, sphenoid and ethmoid sinuses, are mainly located in the maxillary sinus (MS). Each sinus is lined by a ciliated pseudostratified epithelium that is interspersed with mucus-secreting goblet cells. The mucosal surface of the MS is covered with the developed mucociliary epithelium in the airway epithelium that clears inhaled pathogens. ${ }^{1}$ The nasal microvilli increase in surface area to retain moisture and to prevent drying of the surface. ${ }^{2}$ Moreover, pathological data have indicated congestion, submucosal oedema, retention cysts, true polyps and inflammation in the mucosa of the maxillary sinus, such as eosinophilia, basement membrane thickening, goblet cell hyperplasia and dystrophic calcification., ${ }^{1,3}$ However, the structural cilia of the nasal sinus remain poorly understood. The thickness of the nasal Schneiderian membrane (SM) of the MS is also an important landmark for dental implant surgery. Moreover, little is known about promoter immunohistochemical markers of the SM of human maxilla samples. Thickening of the mucosa of the MS was observed in patients undergoing sinus lift treatments, which is a risk factor for MS excretory dysfunction (Manji et al., 56.5\%; Ritter et al., 38\%).,5 Cone-beam computed tomography (CBCT) analysis is a useful tool for sinus lift dental treatment. ${ }^{6}$ In previous reports, the thickness of the maxillary mucosa was measured using a CBCT apparatus or magnetic resonance imaging. ${ }^{4,5}$, However, a difference in SM thickness between CBCT analysis and histological observation was reported ${ }^{89}$ Age, but not sex, is also an important factor that influences sinus membrane thicknesses measured using CBCT analysis, ${ }^{10}$ and periodontal bone loss may be related to MS lateral bone wall thickness. ${ }^{11}$ Therefore, we investigated in detail the relationship between CBCT analyses and histochemical observations of SM in the human maxilla. Calcitonin gene-related peptide (CGRP) is involved in vasodilation and interacts with the sympathetic nervous system in the periphery. Antidromic stimulation of the rat trigeminal ganglion and subsequent neurogenic inflammation affect the expression of neurotrophin receptors in retinal cells by stimulating the trigeminal ganglion to trigger the release of substance $\mathrm{P}$ and CGRP from the sensory nerve terminals of C-fibres. ${ }^{12}$ Substance $\mathrm{P}$ and CGRP are associated with pain and inflammation and are located in the MS at macroscopic levels. ${ }^{13}$ Moreover, CGRP-positive nerve fibres have been observed in the SM and are located around large blood vessels in the lamina propria. In the human trigeminal ganglion (TG), immunohistochemical staining for CGRP is more commonly observed in TG neurons. ${ }^{14}$ Moreover, blood flow is controlled by sympathetic and parasympathetic nerves. In addition, CGRP controls smooth muscle by reducing blood flow, which restricts the blood supply to the alveolar bone. In contrast, Li et al. reported the presence of regenerating nerve fibres expressing CGRP at the fracture callus in close proximity to chondrocytes in experimental mouse tibia fractures. ${ }^{15}$ CGRP is an important marker of bone formation. In contrast, dental treatment at these sites has not focused on the bone dynamics on the side of the SM of the maxilla, and the presence of the nasal floor side also does not require special attention in sinus lift treatments. However, pain in this area is also relevant to patient discomfort and recovery after dental treatment. We investigated the expression of CGRP to simultaneously evaluate pain and bone and mucosa dynamics. Various SM cells may be controlled by the CGRP-positive ganglion cells of the TG. Therefore, we aimed to investigate the detailed morphological characteristics of the maxilla by performing CBCT and observing CGRP expression in the human maxilla and TG. These morphological features of the maxilla and SM were evaluated using principal component analysis (PCA). CGRP distributions influence the blood vessel supply system and pain in the maxilla, and our study provides useful baseline data for the risk of damage to the preoperative MS after surgical operations.

\section{Materials and Methods}

\section{Sample preparation}

One hundred twenty-six maxilla ( 71 cadavers; male, $n=26$; female, $n=45$ ) that were preserved at Tokyo Medical University and Nippon Dental University were used in this study; the cadavers ranged in age from 64 to 104 years (mean: $82.3 \pm 10.1$ years). All specimens were selected for our study based on the medical record of each deceased individual. All human cadavers used in the study were donated for dissection. The samples were injected with $10 \%$ formalin with return perfusion via the femoral artery.

\section{CBCT scanning}

Images of the human cadavers were acquired using a CBCT device (AZ 3000CT; Asahi Roentgen Industry, Kyoto, Japan). Images of the MS and surrounding structures of the maxilla were also acquired from samples of the premolar and molar regions. CBCT images were obtained using a tube potential of $85 \mathrm{kV}$ and tube current of $4 \mathrm{~mA}$, and the scans encompassed cylindrical areas of $79 \varphi \times 71 \mathrm{~mm}$ at high resolution (i.e., voxel size $=0.155 \mathrm{~mm}$ ). Measurements of the maxillary elements of the SM were collected from the three-dimensional CBCT images using NEOPREMIUM (Asahi Roentgen Industry, Kyoto, Japan) and Micro AVS ver. 11 software (KGT Industry, Tokyo, Japan). The median sagittal plane and Frankfort plane positioned perpendicular to the floor were defined as the reference planes, and then the SM focus was the centre for the axial first molar teeth as the measurement position.

\section{Measurement points and identification of each maxilla}

Eight elements were measured in the human MS: the maximum width of the MS (WMS), maximum height of the MS (HMS), thickness of the anterior region of the MS floor bone (AMB) and mucosa (AMM) at the border of the MS floor, thickness of the middle region of the bone (MMB) and mucosa (MMM) in the MS floor, and thickness of the posterior region of the bone (PMB) and mucosa (PMM) of the MS floor at the border of the MS floor (Figure 1). For PCA, we analysed the tooth number, age, sex and difference between the right and left sides (LR). The median sagittal plane and Frankfort plane were defined as reference planes (Figure $1 \mathrm{~A}-\mathrm{D}$ ). We defined the infrazygomatic crest of the edentulous specimens and missing first molar samples, and existing first molar samples were explored at the centre of the first molars. The lower end of the anterior bony wall and the lower end of the posterior bony wall that related to alveolar bone of the MS were divided into three equal parts, and measurements were taken mesiodistally along the central part of each length where bone matrices were clearly defined. Two anatomical and surgical specialists defined and obtained all measurement data 4 times under the suggestion of a radiology specialist.

\section{Immunohistochemical staining for CGRP and CD31 in paraffin-embedded sections}

The molar region of the maxilla and TG of each cadaver were used for immunohistochemical staining. Decalcified and calcified specimens were prepared and used for analyses. The samples were decalcified with $10 \%$ EDTA-PBS for $21-36$ days at $4{ }^{\circ} \mathrm{C}$ and then washed with PBS for $30 \mathrm{~min}$. The samples were fixed with $70 \%$ alcohol and were then dehydrated by the normal method. The TG and maxilla containing the SM were fixed with tissue fixative (Genostaff Co., Ltd., Tokyo, Japan), embedded in paraffin and cut into $5-\mu \mathrm{m}$ sections. The sections, which were cut vertically from 

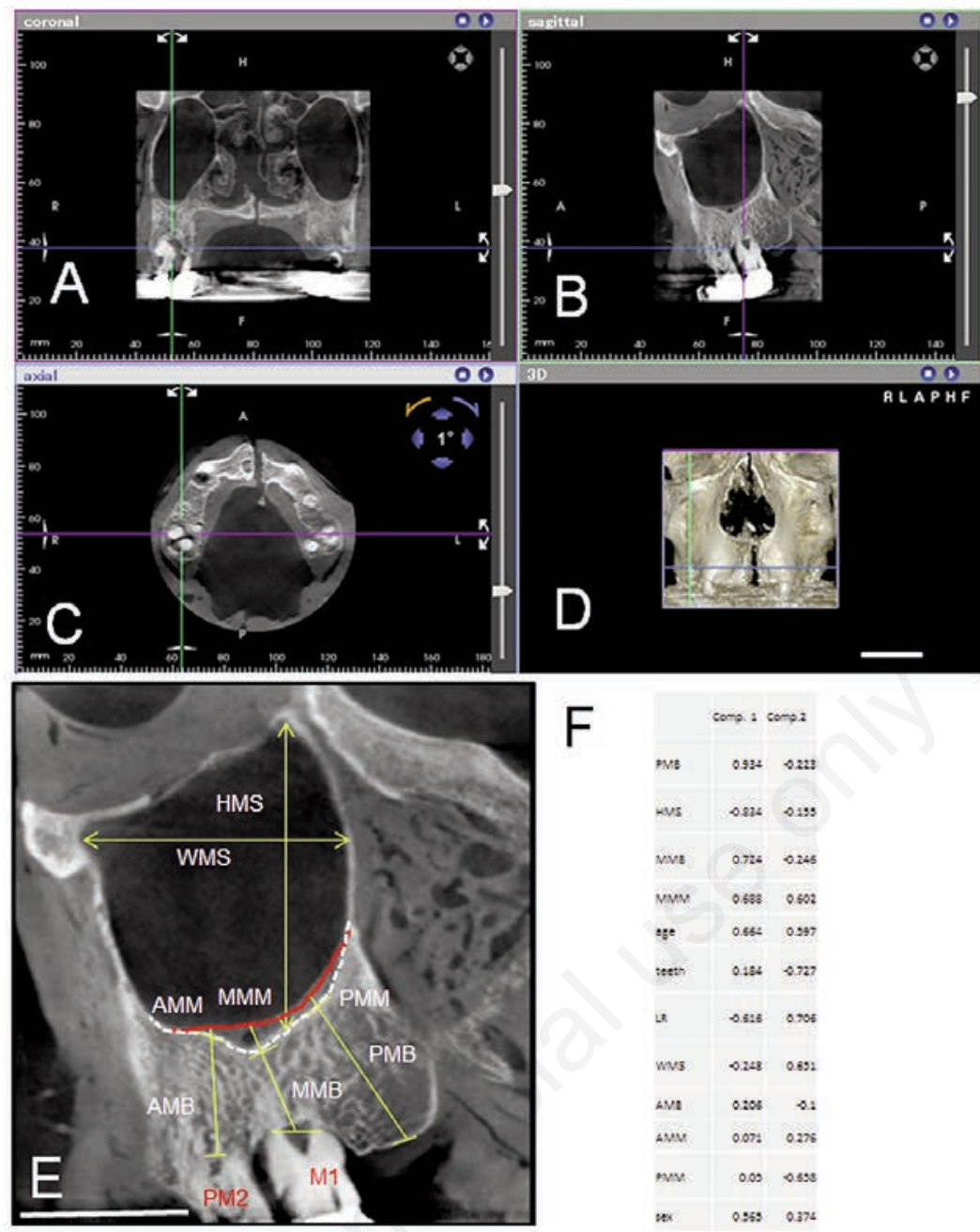

F $\cos 2 x \cos \theta$

pus o.934 ouz

the 0 one

vve ore ous

NWN $0.688 \quad 0.502$

sge $0.565 \quad 0.997$

seeth 0.34 orn?

u. $0.628 \quad 0.706$

wus $\quad 0.248 \quad 0.692$

ave 0.206 o.

AMv $0.075 \quad 0.276$

pure 0.02 osss

nex $0.58 \quad 0.374$

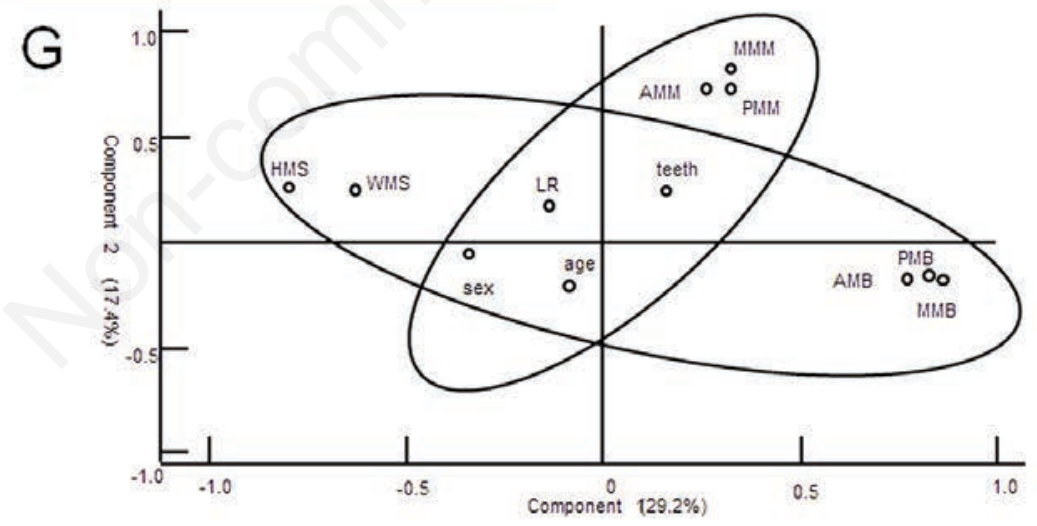

Figure 1. Positions of the maxillary sinus measured in this study. Positions of the maxillary sinus (MS) and maxilla measured in the coronal (A), sagittal (B) and axial sections $(C)$ (voxel size $=0.155 \mathrm{~mm})$ and the $3 \mathrm{D}$ structure $(\mathrm{D})(79 \phi \times 71 \mathrm{~mm})$ that were obtained using a CBCT apparatus (AZ 3000CT). The measurement points are shown as green crosses, and blue lines show the position of the first molar region. (E) Eight measured positions in the maxilla are shown. The maximum width of the MS (WMS), maximum height of the MS (HMS), thickness of the anterior region of the MS floor bone (AMB) and mucosa (AMM) at the border of the MS floor, thickness of the middle region of the bone (MMB) and mucosa (MMM) in the MS floor, and the thickness of the posterior region of the bone (PMB) and mucosa (PMM) of the MS floor at the border of the MS floor; PM2, premolar teeth; M1, first molar teeth; white dotted lines show the border of the MS floor of the maxilla; and red lines show the border of Schneiderian membrane: scale bars: A-E) 20 mm. F) Matrices of component $1(\mathrm{Com} .1)$ and component $2(\mathrm{Com} .2)$ from the CBCT analysis and measurement data obtained from the human maxilla. G) PCA of the components: two large groups were classified into components 1 and 2. Eight measured data points and four elements were used: age, sex, difference between left and right (LR) and number of teeth (teeth) in the maxilla based on CBCT images. Eight elements were analysed in the maxilla: the maximum width of the MS (WMS), maximum height of the MS (HMS), thickness of the anterior region of the MS floor bone (AMB) and mucosa (AMM) at the border of the MS floor, thickness of the middle region of the bone (MMB) and mucosa (MMM) in the MS floor, and thickness of the posterior region of the bone (PMB) and mucosa (PMM) of the MS floor at the border of the MS floor. 
the tooth axis to the lip, were deparaffinized with xylene and dehydrated through treatment with a series of ethanol solutions in phosphate-buffered saline. Endogenous peroxidase was blocked with $0.3 \% \mathrm{H}_{2} \mathrm{O}_{2}$ in methanol for $30 \mathrm{~min}$, and the sections were then blocked with Protein Block (G-Block, Genostaff Co., Ltd., Tokyo, Japan) and an avidin/biotin blocking kit (Vector SP-2001, Vector Laboratories, Inc. Burlingame, CA, USA). Samples were incubated overnight at $4^{\circ} \mathrm{C}$ with primary rabbit polyclonal antibodies against CD31 (1:1,000; anti-CD31 mouse mAb M0823, Dako A/S, Glostrup, Denmark) and calcitonin gene-related peptide (CGRP) (1:1,000; ab47027, Abcam, Cambridge, UK) or normal goat serum as the negative control (other serial sections). The sections were washed with Tris-buffered saline, $\mathrm{pH} 7.5$, containing $0.05 \%$ nonionic surfactant (Tween-20) (TBST) and Tris-buffered saline (TBS) (0.05 M TBS, $0.05 \%$ Tween 20, pH 9.0) and were incubated with biotin-conjugated goat anti-rabbit IgG (E0432, Dako) at room temperature for $30 \mathrm{~min}$. The sections were washed with TBST and TBS and incubated with peroxidase-conjugated streptavidin (Nichirei, Tokyo, Japan) at room temperature for $5 \mathrm{~min}$. Peroxidase activity was visualized using diaminobenzidine. The sections were counterstained with Mayer's haematoxylin (MUTO Pure Chemicals Co., Ltd. Tokyo Japan), dehydrated, and mounted with malinol (MUTO Pure Chemicals Co., Ltd.). Each serial section was also stained with haematoxylin and eosin. Images were acquired using a microscope (Leica DM 2500 16; Leica Microsystems, Buffalo Grove, IL, USA) and Leica Application Suite software (Leica Microsystems). For rate measurements, each cell and blood vessel were randomly selected in the maxilla and TG. We also analysed the reaction in each section using image analysis software (WinROOF, MITANI Co., Tokyo, Japan), which was based on the measurement methods reported by Mitsuoka et al. ${ }^{16}$ with some modifications. The average positive intensity (OD) was defined as weak $(>1,999 \mathrm{OD}=1+)$, moderate $(2,000-6,999$ $\mathrm{D}=2+)$, or strong $(7,000 \mathrm{OD}<=3+)$ positive staining based on the percentage (Figure 2).

\section{Statistical analysis}

The measured data were analysed using two-way analysis of variance (ANOVA) followed by Bonferroni's post hoc test with one categorical independent variable and one continuous variable (the independent variable was the group). Pearson's correlation coefficients were calculated to determine the correlations. The differences in measured data between females and males and between the dentulous and edentulous groups were analysed using the Student's $t$-test. The level of significance was set to $\mathrm{p}<0.05$. Multivariate modelling in PCA was used to estimate the interactions of the measured $\mathrm{CBCT}$ data and the differences between the left and right (LR) sides, age, and sex. We performed clustering analyses using the average linkage between groups (hierarchical clustering analysis algorithm) based on the significant components of PCA performed on individuals. ${ }^{17}$ All statistical analyses were performed using IBM SPSS Statistics Base version 22 (IBM Corporation, New York, USA).

\section{Results}

\section{Measurements of the maxillary sinus}

The measurement data for the 8 elements of the MS are shown in Table 1. For measurements of SM thickness, the posterior region of the MS floor was the largest (total, $0.97 \pm 0.16 \mathrm{~mm}$; male, $2.47 \pm 0.43 \mathrm{~mm}$; dentulous, $1.12 \pm 0.18 \mathrm{~mm}$ ). The thicknesses of alveolar bone from the MS floor to the palatine side of the maxillary region were measured, and the posterior region was thicker than the other regions (total, average $11.41 \pm 0.48 \mathrm{~mm}$; male, average $12.45 \pm 0.85 \mathrm{~mm}$; female, average $10.81 \pm 0.56 \mathrm{~mm}$; and edentulous, average $11.05 \pm 0.66 \mathrm{~mm}$ ). Significant differences in the WMS (male, average 29.0 \pm 0.95 ; female, average $30.97 \pm 0.58, \mathrm{p}<0.05$ ) and HMS (male, average $28.7 \pm 1.10$; female, average $33.05 \pm 0.80$, $\mathrm{p}<0.05$ ) were observed between samples from males and females (Table 2).

\section{Correlation coefficients between measured elements from CBCT data}

The twelve elements measured in the human maxilla using CBCT revealed significant correlations between $\mathrm{PMB}$ and $\mathrm{MMB}$ $(\mathrm{r}=0.741, \mathrm{p}<0.01)$; AMB and MMB $(\mathrm{r}=0.725, \mathrm{p}<0.01)$; PMM and MMM (r=0.667, $\mathrm{p}<0.01)$; HMS and WMS $(\mathrm{r}=0.657, \mathrm{p}<0.01)$ and MMM and AMM $(\mathrm{r}=0.655, \mathrm{p}<0.01)$. Other significant correlations are shown in Table 2.

\section{PCA for human maxilla at measured points, ageing, sex, tooth number, and CBCT data}

PCA was performed for 12 measured elements in the maxilla, which were plotted in a two-dimensional space that was defined by the two axes of component 1 (x-axis) and component 2 (y-axis) (Figure 3A). The two principal components significantly explained $29.2 \%$ (component $1,29.2 \%$ and component $2,17.4 \%$ ) of the information in the maxilla dataset. Three elements (AMM, MMM, PMM and number of teeth) positively contributed, and two elements (sex and age) negatively contributed to the variance in component 1. The positive contributions of three elements (WMS, HMS and difference between right and left) and negative contributions (AMB, MMB, and PMB) explained the variance in component 2 (Figure 3A). The contributions of 4 measured elements (PMB, 0.934; HMS, -0.834; MMB, 0.724; MMM, 0.688; and age, 0.664 ) explained the variance in component 1 . A positive contribu-

Table 1. Sites measured in the maxillary sinus and maxilla using CBCT. Eight elements were measured in the human MS: the maximum width of the MS (WMS), maximum height of the MS (HMS), thickness of the anterior region of the MS floor bone (AMB) and mucosa (AMM) at the border of the MS floor, thickness of the middle region of the bone (MMB) and mucosa (MMM) in the MS floor, and thickness of the posterior region of the bone (PMB) and mucosa (PMM) of the MS floor at the border of the MS floor (see Figure 1). Significant differences were found in the WHS and HMS between females and males.

\begin{tabular}{lccccccccc} 
& Age & AMIM & MIMIM & PMIM & WIMS & HIMS & AMIB & MIMIB & PMIB \\
Male ( $\mathrm{n}=48)$ & $77.60 \pm 1.2$ & $1.67 \pm 0.18$ & $1.69 \pm 0.21$ & $2.47 \pm 0.43$ & $29.0 \pm 0.95$ & $28.72 \pm 1.10$ & $11.37 \pm 0.81$ & $9.68 \pm 0.68$ & $12.45 \pm 0.85$ \\
Female ( $\mathrm{n}=78)$ & $86.70 \pm 1.08$ & $1.70 \pm 0.18$ & $1.84 \pm 0.21$ & $1.84 \pm 0.18$ & $30.97 \pm 0.58^{*}$ & $33.05 \pm 0.80^{*}$ & $9.31 \pm 0.66$ & $7.74 \pm 0.52$ & $10.81 \pm 0.56$ \\
\hline Dentulous $(\mathrm{n}=74)$ & $79.75 \pm 1.14$ & $0.67 \pm 0.14$ & $0.98 \pm 0.20$ & $1.12 \pm 0.18$ & $31.01 \pm 0.67$ & $32.2 \pm 0.94$ & $12.11 \pm 0.68$ & $8.75 \pm 0.61$ & $11.74 \pm 0.68$ \\
Edentulous ( $\mathrm{n}=52)$ & $86.97 \pm 1.23$ & $0.765 \pm 0.17$ & $0.88 \pm 0.18$ & $0.80 \pm 0.27$ & $26.90 \pm 1.20$ & $30.53 \pm 0.83$ & $7.90 \pm 0.69$ & $8.11 \pm 0.58$ & $11.05 \pm 0.66$ \\
Total $(\mathrm{n}=126)$ & $83.3 \pm 0.90$ & $0.71 \pm 0.11$ & $0.93 \pm 0.14$ & $0.97 \pm 0.16$ & $29.0 \pm 0.70$ & $31.4 \pm 0.63$ & $10.10 \pm 0.52$ & $8.45 \pm 0.42$ & $11.41 \pm 0.48$
\end{tabular}




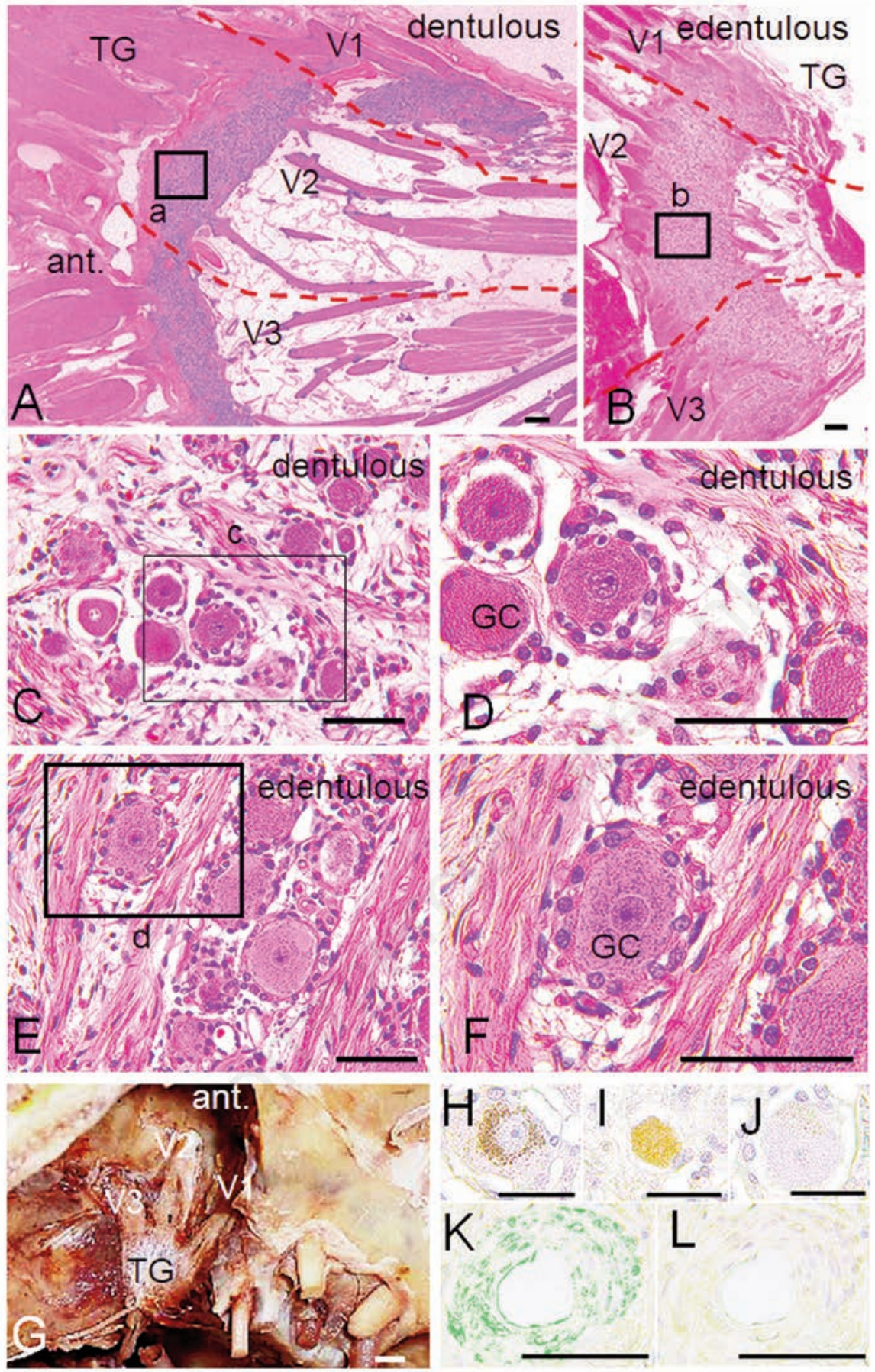

Figure 2. Haematoxylin-eosin (HE)double staining in sagittal sections of the trigeminal ganglion and ganglion cells classified based on CGRP-positive reactions. In the human trigeminal ganglion (TG), which is composed of numerous fibres and ganglion cells, large ganglion cells (GCs) around numerous connective tissue fibres accumulate at the anterior border of the TG. Edentulous samples (B, E, F) contain smaller structures than dentulous samples (A, C, D). A) HE staining in sagittal sections of dentulous samples. B) HE staining in the sagittal sections of edentulous samples. C) Images presented in the small squares (a) in panels A and D. D) Higher magnifications of the images presented in panel C (square c); the large ganglion with a central nucleus is located around some satellite cells; many axon fibres display an irregular trajectory. E) Higher magnification of the image shown in panel B (square b); large ganglion cells are located around regularly arranged axon fibre bundles. F) Higher magnification of the image shown in panel E. G) Macroscopic view of the trigeminal ganglion (TG) at the base of the internal cranium. The red dotted line shows the border between the V1 (ophthalmic nerve), V2 (maxillary nerve), and V3 (mandibular nerve) zones in the TG; ant, anterior direction. H-L) The ganglion cells are classified based on CGRP-positive reactions: images of immunohistochemical staining showing anti-CGRP-positive ganglion cells in the human trigeminal ganglion (TG). H) Strong anti-CGRP-positive reaction in the centre of ganglion cells. I) Moderate anti-CGRP-positive reaction in whole ganglion cells. J) Weak anti-CGRP reaction on the lateral side of ganglion cells; these reaction levels were classified as follows: the average positive intensity (OD) was defined based on the percentage as weak $(>1,999 \mathrm{OD}=1+$, density described by Mitsuoka $e t$ al. in 2018), moderate $(2,000-6,999 \mathrm{D}=2+)$, or strong $(7,000 \mathrm{OD}<=3+)$ positive staining. $\mathrm{K}-\mathrm{L})$ The algorithm also provided a green markup image for the visualization of a positive reaction. $K$ ) The algorithm provided a green colour for the visualization of positive reactions. L) Anti-CGRP positive reaction. Scale bars: A-F, H-L) $50 \mu \mathrm{m}$; G) $5 \mathrm{~mm}$ 
tion of 2 measured elements (MMM 0.602; age, 0.597; LR, 706; WMS, 0.651) and a negative contribution (number of teeth, 0.727 ) to the variance in component 2 were noted (Figure $1 \mathrm{G}$ ).

\section{Histology of the trigeminal ganglion}

In the macroscopic observations, the TG at the base of the internal cranium was composed of the ophthalmic nerve, maxillary nerve, and mandibular nerve. The TG is composed of large sensory neurons and numerous small satellite cells that are covered by collagenous fibre capsules. The TG of the edentulous samples contained abundant connective tissue. Sensory ganglion cells of the edentulous samples were observed between developed fibre bundles; in contrast, the sensory ganglion cells of the dentulous samples were mainly located in the TG (Figure 2).

\section{Localization of the anti-CGRP-positive reaction in the trigeminal ganglion and maxilla}

In the human TG, the strong anti-CGRP-positive reaction in the centre of the ganglion cell, moderate anti-CGRP-positive reaction in the whole ganglion cell, and weak anti-CGRP reaction in the lateral side of the ganglion cells were classified in the TG (Figure 1). More anti-CGRP-positive cells were observed in the dentulous samples than in the edentulous samples (Figure 3 A-D). The TG cells in the maxillary nerve area (V2; Figure 2) of the dentulous samples were composed of anti-CGRP-positive cells (strong, average 3.8 $\pm 0.63 \%$; moderate, average $4.9 \pm 0.90 \%$; weak, average $68.9 \pm 10.7 \%$; and no reaction cells, average $22.4 \pm 3.13 \%$ ). In contrast, the TG cells in the edentulous samples were composed of anti-CGRP-positive cells (strong, average $6.1 \pm 1.49 \%$; moderate, average $5.8 \pm 1.42 \%$; weak, average $39.3 \pm 3.49 \%$ ) and cells with no reaction (average $48.8 \pm 2.62 \%$ ) ( $n=50$ cells per sample) (Figure 3 A-D). Moreover, anti-CGRP-positive reaction cells in dentulous samples was more appeared compared to that of edentulous samples in the maxilla (Figure $3 \mathrm{E}-\mathrm{H}$ ). In the macroscopic observations, the mucosa of the MS and maxillary alveolar bone were clearly defined in the maxilla in the sagittal sections (Figure 3I). The SM of the MS was composed of numerous ciliated and goblet cells with an orderly arrangement in the upper region of the maxilla. The developed sol layer also contained numerous goblet cells in the SM (Figure $3 \mathrm{~K}-\mathrm{L}$ ). Mucus was also observed on the sol layer of the SM in the region of the MS side. In the submucosal region, the submucosal capillary bed was composed of developed blood vessels and small nerves (Figure $3 \mathrm{~J}-\mathrm{N}$ ). CGRP-positive blood vessels were observed in the connective tissue beneath the MS in the edentulous samples, in contrast to CGRP-positive reactions, which were mainly found around nerves at the alveolar bone in dentulous samples (Figure $3 \mathrm{M}-\mathrm{P}$ ). On the MS side of the maxilla, CGRP-positive signals were detected primarily in the basal mucosal cells along with goblet and ciliated cells, blood vessel walls, and osteoblasts in the cortical and trabecular bones. Signals were also identified in the epineurium, perineurium, and endoneurium of the nerve (Figure $3 \mathrm{O}-\mathrm{R}$ ).

\section{Localization of CGRP-positive signals in the blood ves- sels and nerves located in the mucosa and bone of the MS}

CGRP- and CD31-positive immunohistochemical reactions $(+)$ were clearly detected in the sagittal sections around the blood vessels and small nerves in the submucosal region of the human maxilla on both the maxillary and palatine sides. A CGRP-positive reaction was also observed in osteocytes in the small bone cavities (Figure $4 \mathrm{~A}-\mathrm{T}$ ). In the $\mathrm{SM}$ of palatine, a CGRP-positive reaction was observed in small epithelial cells, as shown in Figure 4A. Scattered CGRP-positive osteocytes were observed in alveolar bone (Figure 4D). CGRP-positive blood vessels and small nerves were also observed in the submucosal layer (Figure 4B). CGRPpositive blood vessels and osteocytes were also noted at the border of the alveolar bone and submucosal layer (Figure 4C).

\section{Comparison of the levels of anti-CGRP-positive signals in blood vessels between the dentulous and edentulous groups}

We analysed the levels of anti-CGRP-positive signals in the blood vessels in four regions: the MS side beneath the SM layer, bone layer of the MS side, palatine side beneath the layer of the $\mathrm{SM}$, and bone layer of the palatine side. The long diameters of the lumen of the anti-CGRP-positive blood vessels in the dentulous group (bone: maxillary side, average $40.8 \pm 5.2 \mathrm{~mm}$; palatine side, average $25.9 \pm 3.2 \mathrm{~mm}$; mucosa member: maxillary side, average $45.0 \pm 7.9 \mathrm{~mm}$; and palatine side, average $33.68 \pm 4.6 \mathrm{~mm}$ ) were greater than those in the edentulous group (bone: maxillary side, average $25.2 \pm 3.0 \mathrm{~mm}$; palatine side, average $32.7 \pm 2.6 \mathrm{~mm}$; mucosa member: maxillary side, average $30.6 \pm 2.8 \mathrm{~mm}$; and palatine side, average $26.0 \pm 1.7 \mathrm{~mm}$ ). The densities of the anti-CGRP-

Table 2. Correlation coefficients among the elements that were measured from CBCT data. The eight elements that were measured for the human maxilla using CBCT analysis exhibited significant correlations (see Table 1 for the abbreviations).

\begin{tabular}{|c|c|c|c|c|c|c|c|c|c|c|c|c|}
\hline & Age & LR & Sex & Teeth & AMM & MMM & PMM & WMS & HMS & BTA & BTM & BTP \\
\hline Age & 1 & & & & & & & & & & & \\
\hline RL & & 1 & & & & & & & & & & \\
\hline Sex & $0.436^{* *}$ & & 1 & & & & & & & & & \\
\hline Teeth & $-0.407^{* *}$ & & & 1 & & & & & & & & \\
\hline AMM & & & & & 1 & & & & & & & \\
\hline MMM & & & & & $0.655^{* *}$ & 1 & & & & & & \\
\hline PMM & & & & & $0.394^{* *}$ & $0.667^{* *}$ & 1 & & & & & \\
\hline WMS & & & & & & & & 1 & & & & \\
\hline HMS & & & & $0.280^{* *}$ & $0.191^{*}$ & & & $0.657^{* *}$ & 1 & & & \\
\hline AMB & & & & & $0.371^{* *}$ & & & $-0.329 * *$ & $-0.517^{* *}$ & 1 & & \\
\hline MMB & & & & $-0.199 *$ & & & & $-0.402 * *$ & $-0.644^{* *}$ & $0.725^{* *}$ & 1 & \\
\hline PMB & & & & & & & & $-0.417 * *$ & $-0.602^{* *}$ & $0.649 * *$ & $0.741^{* *}$ & 1 \\
\hline
\end{tabular}

${ }^{*} \mathrm{p}<0.05 ;{ }^{*} \mathrm{p}<0.01$ 
positive signals in the blood vessels of the dentulous group were higher (bone: palatine side, average $8.48 \pm 0.91 \%$; mucosal layer: maxillary side, average $8.48 \pm 0.53 \%$; and palatine side, average $7.28 \pm 0.87 \%$ ) than those in the edentulous group (bone: palatine side, average $2.39 \pm 0.40 \%$; mucosal layer: maxillary side, average
$2.02 \pm 0.58 \%$; and palatine side, average $3.34 \pm 0.38 \%$ ), with the exception of the maxillary side (dentulous, average $4.30 \pm 0.87 \%$ and edentulous, average $4.41 \pm 0.68 \%$ ) ( $n=50$ blood vessels per group) (Figure $3 \mathrm{E}-\mathrm{H}$ ).

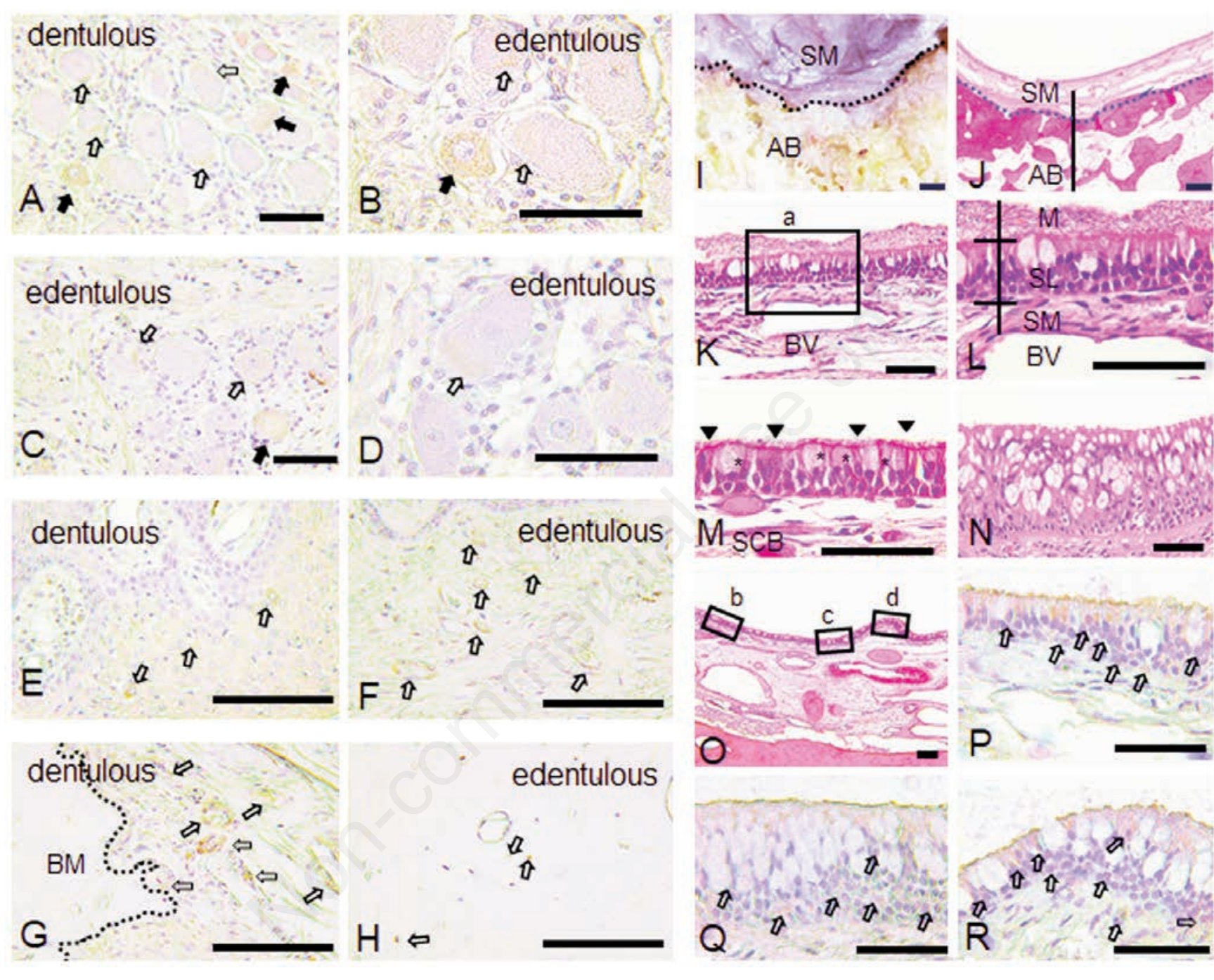

Figure 3. Locations of anti-CGRP-positive signals in the human maxilla and trigeminal ganglions between dentulous and edentulous samples and the Schneiderian membrane (SM) in the human maxillary sinus. The relatively strong positive reactions for CGRP (black arrows) and moderate reactions for CGRP (white arrows) were observed in the ganglion cells of the dentulous samples in the maxillary nerve area (V2, see Figure 4) (A-B) and are compared to edentulous samples (C-D). Positive signals for CGRP (white arrows) were detected mainly in the blood vessels and around bone matrices in the lamina propria beneath the MS (E-F) and the alveolar bone of the maxilla (G-H). In particular, a strong CGRP-positive signal was observed in blood vessels located at the border of the bone area zone and connective tissue (dot line) of the dentulous samples (G). A relatively strong positive reaction was observed in the small blood vessels of the bone marrow of the edentulous samples (H). B, D) Higher magnifications of the images shown in panels A and C (A, C, E, G: dentulous samples; B, D, F, H, edentulous samples). I-R) Schneiderian membrane. I) Macroscopic observation of the maxilla and SM. J) Haematoxylin-eosin staining of the sagittal sections of the MS floor with alveolar bone. K) Haematoxylin-eosin double staining in sections of the MS showing the thickness of the mucosal layer. L), SM of the MS stained with haematoxylin-eosin in sagittal sections (see panel K-a). M) The goblet cells contained abundant glandular cells located between numerous ciliated cells. N) The mucosa contained numerous goblet cells, and basal cells were observed beneath large blood vessels in the high-magnification image of panel $\mathrm{M}$ (square); numerous developed goblet cells were observed in the SM of the MS. O) Sites at which immunohistochemical staining was observed (c-d) are indicated in this HE stain figure. P) Anti-CGRP-positive cells (white arrows) were identified between numerus goblet cells (see O-b). Q) Anti-CGRP-positive cells (white arrows) were also observed between numerous goblet cells (see 3O-c). R) Anti-CGRP-positive basal cells (white arrows) were located at the basal region of the numerous goblet cells (see $\mathrm{O}$-d); dotted lines show the border of alveolar bone and SM; AB, alveolar bone; $\mathrm{BM}$, bone matrices zone; M, mucus; SM, submucosal layer; SL, sol layer; arrowheads, ciliated cell; * goblet cell; SM, Schneider membrane; SCB, submucosal capillary bed; BV, blood vessel. P-R, white arrows, CGRP-positive cells. Scale bars: I,J) 1 mm; A-R) $100 \mu \mathrm{m}$. 

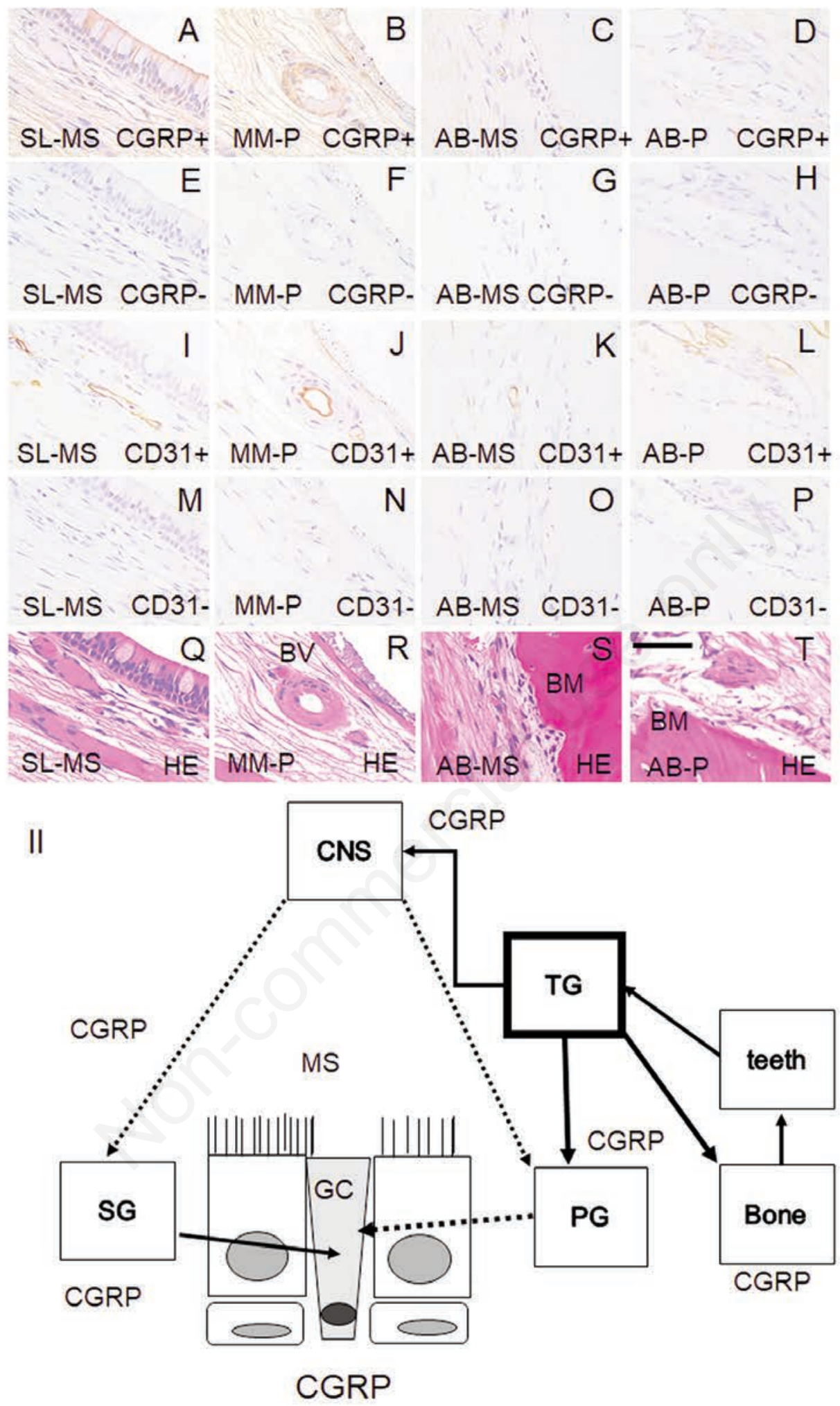

Figure 4. Immunohistochemical staining for CGRP and CD31 on the MS side and oral mucosa side of the maxilla. A, E, I, M, Q) MS side. B, F, J, N, R) Oral mucosa. C, G, K, O, S, Alveolar bone of the MS side. D, H, L, P, T) Alveolar bone of the oral mucosa side. A, B, C, D, Anti-CGRP-positive reactions; I, J, K, L) Anti-CD31-positive reactions. Q, R, S, T) Haematoxylin and eosin staining. E, F, G, H) Negative control for the CGRP reaction; M, N, O, P) Negative control for the CD31 reaction. Scale bar: $100 \mu \mathrm{m}$. II) Schematic of CGRP transport in the mucosal gland of the Schneiderian membrane. Two routes for CGRP transport in the trigeminal ganglion (TG) were identified: CGRP was transported from the axons of sensory nerves to TG cells, and the other route was CGRP delivery to the parasympathetic ganglion from the CNS, in which CGRP affected parasympathetic ganglion cells (pterygopalatine ganglion, PG) and induced acetylcholine transport to the gland. TG cells also deliver CGRP to osteoclast and osteoblast cells of the maxillary bone by tooth stress or tooth loss. BM, bone matrices zone; BV, blood vessels; CNS, central nervous system; GC, goblet cell; MS, maxillary sinus; SG, superior ganglion. 


\section{Discussion}

As the paranasal sinuses are continuous with the nasal cavity, upper respiratory tract infections can spread to the sinuses. Sinus infections (sinusitis) cause inflammation (particularly pain and swelling) of the mucosa. If more than one sinus is affected, the infection is called pansinusitis. Various reports have documented the thickness of the SM by measuring the thickening of the mucosal lining of the sinus in the region adjacent to the involved tooth root. ${ }^{18-20}$ Carmeli et al. indicated that the thickness of the SM ranged from $5 \mathrm{~mm}$ to $10 \mathrm{~mm}$ and that this structure most frequently displayed a circumferential (55.2\%) form. ${ }^{20}$ Mucosal thickening of more than $2 \mathrm{~mm}$ was directly correlated with the presence of MS lesions (opacification, discontinuity of the sinus floor, polypoid lesions, or air-liquid levels). ${ }^{21}$ The thickness of the SM is an important marker of sinusitis. In general, the ability to adsorb dust in the air and expel it as sputum is also impaired, and MS may become more susceptible to inflammation. Mucosal thickening is an important indicator of inflammation, excretory dysfunction, and bone resorption in the maxilla. In our results, the greatest mucosal thickness was mainly observed in the posterior region of the MS floor compared to other regions in both the dentulous and edentulous samples. The posterior region of our measurement site for the alveolar bone contains the roots of the second and third molar teeth. During remodelling of bone matrices, the SM thickness may be affected by the maxilla and palatine bone structures due to the presence of teeth. The mucosal thickness at the floor site of the alveolar bone affects the modelling pressure and balance between the second and third molars or apical lesions near these teeth. According to our PCA data, the SM thickness was also correlated with tooth number; in contrast, a negative correlation was observed between ageing and sex. In contrast, no relationship was observed between the SM thickness and thickness of the alveolar bone in our PCA. Namely, the thickness of the mucous membrane is related to the missing molar teeth. In our measurements of alveolar bone thickness in the sagittal sections using CBCT, the greatest thickness of alveolar bone was observed at the posterior region of the MS floor. The anterior site of the maxillary alveolar bone was thicker in the dentulous samples than in the edentulous samples. Moreover, the dentulous samples of the AMB were thicker than the edentulous samples. Based on these results, components of the alveolar bone at this site were resorbed due to the missing teeth in edentulous samples, despite the lack of a relationship to SM thickness. The alveolar bone structure affected the MS structure in our PCA. These elements of the alveolar bone thickness were negatively correlated with the MS volume. Few histological evaluations of ganglion cells in the trigeminal nerve that control these structures have been performed. CGRP is related to vasodilation and interacts with the sympathetic nervous system to modulate pain and inflammation; CGRP-positive reactions are mainly observed in the blood vessels of human MS. ${ }^{13}$ CGRP is carried from TG neurons through the trigeminal root. ${ }^{22}$ Therefore, we investigated the characteristic features of ganglion cells in the trigeminal nerve. In our results, the compositions of the anti-CGRP-positive signals differed between the dentulous and edentulous samples, and the ratios of positive cells in the dentulous group were higher than those in the edentulous group. For the missing teeth, the mechanical stress of occlusal conditions decreased the amount of alveolar bone due to the lack of tooth root movements, which continued to be controlled by factors that were secreted from the TG cells, such as the CGRP neuropeptide between the terminal sensory nerve system and central nerve system. CGRP is transported through axons from the sensory nerves to TG cells. CGRP of the TG is also delivered to the maxillary bone and affects remodelling in bone matrices, such as positive action on osteoblasts or negative action on osteoclasts. CGRP distribution in the MS can be characterized morphologically, such as by the presence of numerous CGRP-positive goblet cells. CGRP is also mainly released to the bone and goblet cells from the TG by balancing the signal from mastication by the occlusion differences between dentulous and edentulous stress. In addition, CGRP has a positive effect on osteoblasts. ${ }^{23}$ That is, bone thickness levels reflect the condition of teeth in our PCA between alveolar bone measurement data and the presence of teeth. CGRP was also delivered to the sympathetic ganglion, and CGRP affected ganglion cells and goblet cells in the MS. Moreover, SM contains many goblet cells in MS. In general, SM is a mucous secretory unicellular gland that is scattered among multiple rows of ciliated epithelia in the airway mucosa. A triangular core is present at the base. Goblet cells in the conjunctival epithelium secrete mucin. The secreted mucin is a hydrophilic substance. Functionally, airway remodelling is characterized by epithelial thickening, goblet cell hyperplasia, subepithelial basement membrane fibrosis, airway smooth muscle thickening/hyperplasia and narrowing of the lumen due to airway wall thickening. ${ }^{24}$ Therefore, the airway epithelium is mainly composed of ciliated epithelial cells, basal cells, and goblet cells, and the mucus produced by the goblet cells and submucosal glands and the movement of the mucous membranes clear the airway surface. Researchers have speculated that the mucous membrane of the MS also performs the same function, but unlike the general airway, mucus flow occurs from the crescent fissure in the upper part of the MS in a very enclosed space. The mucus of the mucosal membrane is also an extremely important factor in terms of airway surface clearance. In our observations, these goblet cells were regularly arranged between ciliated epithelia, and numerous goblet cells were also observed in parts of the SM of the MS. CGRP has been shown to be involved in glandular secretions, especially goblet cell secretions, in addition to its functions in vascular smooth muscle dilation, bone remodelling, and intrinsic pain. These results show the diverse functions of CGRP. In the nasal mucosa, CGRP affects the blood vessels and sensory nerves in the nasal mucosa. ${ }^{25}$ Substance P, CGRP and vasoactive intestinal peptide display concentration-dependent, but partly separate, vasodilatory profiles in the nasal mucosa. CGRP controls vasodilatation..$^{26,27}$ In general, two pathways for nasal gland secretion have been identified: CGRP-related sensory fibre (C fibre) and the parasympathetic function of vasoactive intestinal polypeptide or acetylcholine. ${ }^{28-30}$ In the nasal mucosa, the transmission pathway of CGRP is the same as that in the maxillary sinus, but upon mucosal thickening, such as nasal congestion due to vasodilation, CGRP distribution in the mucosa is induced by afferent stimulation. Although it can be induced, it is suggested that the MS is a unique site that is also involved in the physical modelling of the bone by a controlled CGRP pathway system. The CGRP distribution and location are related to its secretion by goblet cells in the MS. In particular, an anti-CGRP-positive reaction was observed in some goblet cells and basal cells of the MS. These results suggest that CGRP modulates the production or secretion of these cells. Irie et al. indicated that microvessels expressing CGRP were positively regulated by bone remodelling. ${ }^{31}$ These microvessels are located directly above the root of the first molar, and their adjacent parts are in an important location where the root of the second molar is closer to the MS floor. ${ }^{32}$ The first molars are the most frequently treated implants at the site we observed, and we thought that an analysis of this site was important. Koppe et al. reported a relationship between MS volume and midfacial width in cranial measurements. ${ }^{33}$ Moreover, MS-related elements, such as the WMS and HMS, were larger in female samples than in male samples. Our samples are related to ageing in females with loss of teeth in our samples compared to males. Therefore, when teeth are lost, the alveolar bone structure 
and other structures that are composed of frame-worked maxilla may change or affect the resorption of bone matrices and then enlarge the space of the MS. The percentage of anti-CGRP-positive cells in the dentulous group was higher than that in the edentulous group in our results. Heffner et al. indicated that sensory nerves in the bone adapt to the mechanical environment. ${ }^{34}$ In our study, occlusion changed in response to mechanical stress and resulted in loss of the tooth, which led to bone resorption and finally reduced the sensory nerve response to the reduction in mechanical occlusion due to tooth loss. In the TG, the CGRP-IR neurons that innervated the joint were significantly increased compared to those in normal animals in the acute and chronic phases of arthritis, respectively. ${ }^{35}$ That is, tooth stress may reflect the release of CGRP from the sensory nerve terminals of the MS by the human TG in the same manner. ${ }^{12}$ Therefore, the anti-CGRP reaction was decreased in the alveolar bone of the edentulous group compared to the dentulous group. Moreover, in this study, occlusion was maintained even in the middle defect in the evaluation based on the presence or absence of teeth, so the stimulus was transmitted to the centre, and CGRP was released to the bones and mucous membranes. CGRP controls smooth muscle by reducing blood flow, which restricts the blood supply to the alveolar bone. In general, the blood vessel supply to the alveolar bone and periosteum lamina propria in the MS and palatine have been reported by macroscopic observations. ${ }^{13,36,37}$ Complex blood vessels supply the MS floor of the maxilla, and numerous anti-CGRP-positive vessels have been detected at macroscopic levels. ${ }^{13,38}$ In our results, a strong antiCGRP-positive reaction was observed mainly in the endothelial cell layer, and different reaction levels were detected in the dentulous and edentulous groups at the microscopic level. In particular, a strong anti-CGRP reaction was observed mainly in the large blood vessels of dentulous samples compared to the small blood vessels in the edentulous group. The edentulous maxillae demonstrated decreasing vascularity during bone resorption, and these vascular conditions were related to bone remodelling. In the rat mandible, the percentage of anti-CGRP-positive nerves increased from the surface to the inner region of the alveolar bone. ${ }^{38}$ Therefore, with tooth loss, resorption occurred from the surface region of the alveolar bone and decreased the number of small vessels at this site. Based on the results, vessel size is also an important contributor to the differences between the dentulous and edentulous samples in our study. Finally, our results are important to oral surgeons, especially in cases that require examination of the site of implant placement during dental implant treatment and in the sinus lift procedures that are performed to promote bone formation. These treatments are performed by examining diagnostic images to evaluate the condition of the bone and often do not examine the thickening of soft tissues. Therefore, this research showed that in addition to bone conditions, the presence of neuropeptides such as CGRP, which are markers of pain, and macrodynamics may lead to mucosal thickening and provide clinical information that is related to the prognosis for recovery. The complications in surgical treatments of the SM and the sinus wall were related to the sinus anatomy. ${ }^{39,40}$ Therefore, our information about the SM gives a useful information to the reduce in the occurrence of the complications.

\section{Acknowledgements}

The authors would like to express our greatest appreciation to members of the Department of Anatomy, Tokyo Medical University for their contribution to this research.

\section{References}

1. Beule AG. [Physiology and pathophysiology of respiratory mucosa of the nose and the paranasal sinuses].[Article in German]. Laryngorhinootologie 2010;89:S15-34.

2. Mygind N, Pedersen M, Nielsen MH. Morphology of the upper respiratory epithelium. DF Proctor and Andersen I, Editors. The Nose, upper airway physiology and the atmospheric environment. Amsterdam: Elsevier; 1982. p. 71-97.

3. Coleman H, Meer S, Altini M, Reyneke J, Becker P. Maxillary sinus pathology in 119 patients--a histopathologic study. SADJ 2005; 140:142-5.

4. Manji A, Faucher J, Resnik RR, Suzuki JB. Prevalence of maxillary sinus pathology in patients considered for sinus augmentation procedures for dental implants. Implant Dent 2013;22:428-35.

5. Ritter A, Rozendorn N, Avishai G, Rosenfeld E, Koren I, Soudry E. Preoperative maxillary sinus imaging and the outcome of sinus floor augmentation and dental implants in asymptomatic patients. Ann Otol Rhinol Laryngol 2020;129:209-15.

6. Ata-Ali J, Diago-Vilalta J-V, Melo M, Bagán L, Soldini M-C, Di-Nardo C, et al. What is the frequency of anatomical variations and pathological findings in maxillary sinuses among patients subjected to maxillofacial cone beam computed tomography? A systematic review. Med Oral Patol Oral Cir Bucal 2017;22:e400-9.

7. Munhoz L, Abdala Júnior R, Abdala R, Asaumi J, Arita ES. Diffusion-weighted magnetic resonance imaging in maxillary sinuses inflammatory diseases: Report of three cases and literature review. J Oral Maxillofac Res 2018;9:e4.

8. Monje A, Monje-Gil F, Burgueño M, Gonzalez-Garcia R, Galindo-Moreno P, Wang HL. Incidence of and factors associated with sinus membrane perforation during maxillary sinus augmentation using the reamer drilling approach: A doublecenter case series. Int J Periodontics Restorative Dent 2016; 36:549-56

9. Insua A, Monje A, Chan HL, Zimmo N, Shaikh L, Wang HL. Accuracy of Schneiderian membrane thickness: a cone-beam computed tomography analysis with histological validation. Clin Oral Implants Res 2017; 28:654-61.

10. Zimmo N, Insua A, Sinjab K, Chan HL, Shaikh L, Wang HL. Impact of sex, age, and season on sinus membrane thickness. Int J Oral Maxillofac Implants 2018;33:175-80.

11. Talo Yildirim T, Güncü GN, Colak M, Tözüm TF. The relationship between maxillary sinus lateral wall thickness, alveolar bone loss, and demographic variables: A cross-sectional conebeam computerized tomography study. Med Princ Pract. 2019; 28:109-14.

12. Bronzetti E, Artico M, Kovacs I, Felici LM, Magliulo G, Vignone D, et al. Expression of neurotransmitters and neurotrophins in neurogenic inflammation of the rat retina. Eur $\mathrm{J}$ Histochem 2007;51:251-60.

13. Sato I, Imura K, Miwa Y, Yoshida S, Sunohara M. Distributions of calcitonin gene-related peptide and substance $\mathrm{P}$ in the human maxillary sinus of Japanese cadavers. J Craniomaxillofac Surg 2012;40:e249-52.

14. Azuma Y, Sato I. The localization of calcitonin gene-related peptide in the human trigeminal ganglion and masseter muscle. Okajimas Folia Anat Jpn 2017;93:127-38.

15. Li J, Kreicbergs A, Bergström J, Stark A, Ahmed M. Site-specific CGRP innervation coincides with bone formation during fracture healing and modeling: A study in rat angulated tibia. J Orthop Res 2007;25:1204-12.

16. Mitsuoka K, Kikutani T, Miwa Y, Sato I. Expression of CGRP 
neurotransmitter and vascular genesis marker mRNA is agedependent in superior cervical ganglia of senescence-accelerated prone mice. Neurosci Lett 2018; 664:144-51.

17. Cassar-Malek I, Passelaigue F, Bernard C, Léger J, Hocquette JF. Target genes of myostatin loss-of-function in muscles of late bovine fetuses. BMC Genomics 2007;8:63.

18. Ericson, S., Welander U. Local hyperplasia of the maxillary sinus mucosa after elimination of adjacent periapical osteitis. A follow-up study. Odontol Revy 1966;17:153-9.

19. Hauman CH, Chandler NP, Tong DC. Endodontic implications of the maxillary sinus: a review. Int Endod J 2002;35:127-41.

20. Carmeli G, Artzi Z, Kozlovsky A, Segev Y, Landsberg R. Antral computerized tomography pre-operative evaluation: relationship between mucosal thickening and maxillary sinus function. Clin Oral Implants Res 2011;22:78-82.

21. Sánchez-Pérez A, Boracchia AC, López-Jornet P, Boix-García P. Characterization of the maxillary sinus using cone beam computed tomography. A retrospective radiographic study. Implant Dent. 2016; 25:762-9.

22. Panneton WM, Gan Q. Direct reticular projections of trigeminal sensory fibers immunoreactive to CGRP: potential monosynaptic somatoautonomic projections. Front Neurosci 2014;8:136.

23. Fang Z, Yang Q, Xiong W, Li GH, Liao H, Xiao J, et al. Effect of CGRP-adenoviral vector transduction on the osteoblastic differentiation of rat adipose-derived stem cells. PLoS One 2013;8:e72738

24. Wang J, Dong R, Zheng S. Roles of the inflammasome in the gut liver axis (Review). Mol Med Rep 2019;19:3-14.

25. Stjärne P, Lundblad L, Anggård A, Hökfelt T, Lundberg JM. Tachykinins and calcitonin gene-related peptide: co-existence in sensory nerves of the nasal mucosa and effects on blood flow. Cell Tissue Res 1989;256:439-46.

26. Baraniuk JN, Lundgren JD, Goff J, Mullol J, Castellino S, Merida $\mathrm{M}$, et al. Calcitonin gene-related peptide in human nasal mucosa. Am J Physiol 1990;258:L81-8.

27. Stjärne P, Lacroix JS, Anggård A, Lundberg JM. Compartment analysis of vascular effects of neuropeptides and capsaicin in the pig nasal mucosa. Acta Physiol Scand 1991;141:335-42.

28. Lundberg JM. Peptidergic control of the autonomic regulation system in the orofacial region. Proc Fin Dent Soc 1989; 85:239-50.

29. Kuo HP, Rohde JA, Tokuyama K, Barnes PJ, Rogers DF.
Capsaicin and sensory neuropeptide stimulation of goblet cell secretion in guinea-pig trachea. J Physiol 1990;431:629-41.

30. Matran R. Neural control of lower airway vasculature. Involvement of classical transmitters and neuropeptides. Acta Phsiol 1991;601:1-54.

31. Irie K, Hara-Irie F, Ozawa H, Yajima T. Calcitonin gene-related peptide (CGRP)-containing nerve fibers in bone tissue and their involvement in bone remodeling. Microsc Res Tech 2002;58:85-90.

32. Nimigean V, Nimigean VR, Măru N, Sălăvăstru DI, Bădiţă D, Tuculină MJ. The maxillary sinus floor in the oral implantology. Rom J Morphol Embryol 2008;49:485-9.

33. Koppe T, Weigel C, Bärenklau M, Kaduk W, Bayerlein T, Gedrange T. Maxillary sinus pneumatization of an adult skull with an untreated bilateral cleft palate. J Craniomaxillofac Surg 2006;34:91-5.

34. Heffner MA, Genetos DC, Christiansen BA. Bone adaptation to mechanical loading in a mouse model of reduced peripheral sensory nerve function. PLoS One 2017;12:e0187354.

35. Damico JP, Ervolino E, Torres KR, Sabino Batagello D, CruzRizzolo RJ, Aparecido Casatti C, et al. Phenotypic alterations of neuropeptide $\mathrm{Y}$ and calcitonin gene-related peptide-containing neurons innervating the rat temporomandibular joint during carrageenan-induced arthritis. Eur J Histochem 2012;56: e31.

36. Solar P, Geyerhofer U, Traxler H, Windisch A, Ulm C, Watzek G. Blood supply to the maxillary sinus relevant to sinus floor elevation procedures. Clin Oral Implants Res 1999;10:34-44.

37. Miwa Y, Asaumi R, Kawai T, Maeda Y, Sato I. Morphological observation and CBCT of the bony canal structure of the groove and the location of blood vessels and nerves in the palatine of elderly human cadavers. Surg Radiol Anat 2018;40:199-206.

38. Kudo K, Tanaka K, Ambe K, Kawaai H, Yamazaki S. Immunohistochemical analysis of nerve distribution in mandible of rats. Anesth Prog 2019;66:87-93.

39. Chan HL, Wang HL. Sinus pathology and anatomy in relation to complications in lateral window sinus augmentation. Implant Dent 2011;20:406-12.

40. Testori T, Tavelli L, Yu SH, Scaini R, Darnahal A, Wallace SS et al. Maxillary sinus elevation difficulty score with lateral wall technique. Int J Oral Maxillofac Implants 2020;35:631-8.

Received for publication: 19 February 2021. Accepted for publication: 24 March 2021.

This work is licensed under a Creative Commons Attribution-NonCommercial 4.0 International License (CC BY-NC 4.0).

CCopyright: the Author(s), 2021

Licensee PAGEPress, Italy

European Journal of Histochemistry 2021; 65:3234

doi:10.4081/ejh.2021.3234 